\title{
Von Plastikmüll zu Plastikwertstoffen - Polymerrecycling neu gedacht
}

TILL TISO ${ }^{1}$, HENDRIK BALLERSTEDT ${ }^{1}$, CHRISTIAN EBERLEIN ${ }^{2}$, WOLFGANG ZIMMERMANN ${ }^{3}$, NICK WIERCKX ${ }^{1,4}$, LARS M. BLANK ${ }^{1}$

${ }^{1}$ INSTITUTE OF APPLIED MICROBIOLOGY (IAMB), AACHEN BIOLOGY AND

BIOTECHNOLOGY (ABBT), RWTH AACHEN

${ }^{2}$ UMWELT- UND BIOTECHNOLOGIE, HELMHOLTZ-ZENTRUM FÜR UMWELTFORSCHUNG (UFZ), LEIPZIG

${ }^{3}$ MIKROBIOLOGIE UND BIOVERFAHRENSTECHNIK, INSTITUT FÜR BIOCHEMIE, UNIVERSITÄT LEIPZIG

${ }^{4}$ INSTITUT FÜR BIO- UND GEOWISSENSCHAFTEN - IBG-1: BIOTECHNOLOGIE, FORSCHUNGSZENTRUM JÜLICH

\section{The ever increasing plastic production and the challenges of low} recycling quota, microplastic and plastic in the ocean is summarized as plastic crisis, asking for new technology for end-of-life polymer use. We present a value chain for plastic waste developed in the EU project $\mathrm{P}_{4}$ SB. Plastic is hydrolysed to its monomers by engineered enzymes and the monomers are the feedstock for engineered microbes to synthesise products of value. This upcycling adds incentives for increased polymer recycling.

DOI: $10.1007 / \mathrm{s} 12268-020-1349-7$

(C) Die Autoren 2020
Mit der Entdeckung, großtechnischen Herstellung und Marktetablierung der ersten Kunststoffe in der Mitte des 20. Jahrhunderts setzte der rasante und bisher ungebrochene
Aufstieg der globalen Plastikproduktion ein. Hohes Innovationspotenzial in Industrie und Wissenschaft, vielseitige Einsetzbarkeit der synthetischen Polymere und Eigenschaften

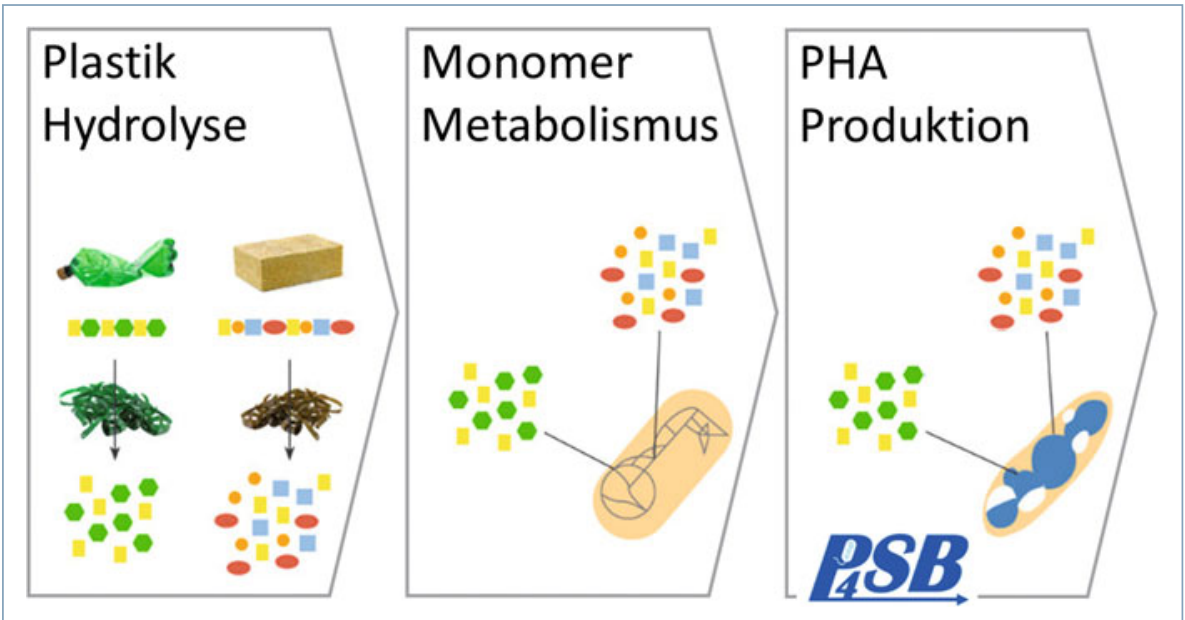

A Abb. 1: Arbeitsablauf von $\mathrm{P}_{4} \mathrm{SB}$ (www.p4sb.eu). Im ersten Schritt werden die Polymere Polyethylenterephthalat (PET) und Polyurethan (PU) enzymatisch gespalten. Daraufhin werden genetisch veränderte Mikroorganismen eingesetzt, um die entstandenen Monomere zu metabolisieren und im dritten Schritt in Biokunststoffe umzuwandeln. wie Leichtigkeit, geringe Toxizität, hohe Festigkeit, Transparenz bis hin zur hohen Haltbarkeit führten zu einer omnipräsenten Nutzung in fast allen Industriezweigen. Jährlich wurden allein im Jahr 2018359 Megatonnen (Mt) Kunststoff produziert. Die sieben wichtigsten Kunststoffpolymere (PVC, PP, PE, PET, PS, PU und PA) machen $92 \%$ aller jemals hergestellten Primärkunststoffe aus (1950-2015: $\sum 8.300$ Mt). Die größten Gruppen sind Polyethylen (PE; 36 \%), Polypropylen (PP; $21 \%$ ) und Polyvinylchlorid (PVC; $12 \%)$, gefolgt von Polyethylenterephthalat (PET), Polyurethan (PU) und Polystyrol (PS) (je $10 \%$ ). Es wird geschätzt, dass $30 \%$ aller jemals hergestellten Kunststoffe derzeit noch genutzt werden, $12 \%$ verbrannt, $9 \%$ recycelt und nur $0,9 \%$ mehr als einmal wiederverwendet wurden. Der Hauptanteil mit rund 4.900 Megatonnen wird in Deponien gelagert oder ist aufgrund seiner Langlebigkeit ubiquitär in der terrestrischen und marinen Umwelt verteilt [1, 2]. Selbst wenn Kunststoffe in der Umwelt zerfallen oder abgebaut werden, sind sie sehr lange als kleinere, für das bloße Auge unsichtbare Teile (Mikrokunststoffe) vorhanden, die das Meeresleben beeinflussen und sich in der Nahrungskette ausbreiten können [3]. Obwohl das Bewusstsein für die negativen Auswirkungen von Plastik in der Umwelt wächst, erleben wir ein noch immer ungebrochenes Wachstum der vor allem aus fossilen Rohstoffen synthetisierten Kunststoffe. Ein Lösungsansatz könnte eine Kreislaufwirtschaft mit annähernd $100 \%$ recycelbaren, langfristig verwendeten Kunststoffen sein. Verschiedene gesellschaftliche Initiativen von NGOs (non-governmental organizations), der Europäischen Kommission bis hin zu Unternehmen (z. B. Pacific Garbage Screening) versuchen hier, neue Lösungen zu entwickeln. Ein erster Schritt auf dem Weg in eine Kreislaufwirtschaft ist der Lösungsansatz Abfall aus herkömmlichen, minderwertigen Kunststoffen, die nicht direkt recycelt werden können, als Substrat für die mikrobielle Produktion hochwertiger, biologischer Polymere, wie z. B. Polyhydroxyalkanoate (PHA), zu nutzen [4]. Dieses biologische Upcycling war das Thema 
des von der EU geförderten Projektes $\mathrm{P}_{4} \mathrm{SB}$ (From plastic waste to Plastic value using Pseudomonas putida Synthetic Biology).

Für das neuartige Upcycling wurden in $\mathrm{P}_{4}$ SB PET- und PU-hydrolysierende Enzyme für die Depolymerisation dieser Kunststoffe $\mathrm{zu}$ ihren monomeren Komponenten entwickelt und eingesetzt. Recycling von PET ist durch hohen Energiebedarf, Materialverlust und unvollständige Rückgewinnung der Monomere (Ethylenglykol [EG] und Terephthalat [TA]) eingeschränkt. Im Gegensatz zu PET ist PU in Bezug auf die Monomerzusammensetzung äußerst vielfältig. Da es sich bei PU meist um Duroplaste handelt, die nicht eingeschmolzen werden können, werden PUs fast nicht rezykliert.

Für den zweiten katalytischen Schritt werden rekombinante Pseudomonas putidaStämme aufgebaut, die Stoffwechselwege zur effizienten Umwandlung der Polymermonomere zu zentralen Metaboliten des bakteriellen metabolischen Netzwerks besitzen. Diese neuartigen Stämme werden dann für die Produktion des biologisch abbaubaren Polymers PHA genutzt (Abb. 1).

\section{Enzymatische Aufspaltung der Polymere}

Mikrobielle Polyesterhydrolasen, die in der Lage sind, amorphes oder niedrigkristallines PET bei Temperaturen in der Nähe der Glasübergangstemperatur $\left(60-70{ }^{\circ} \mathrm{C}\right)$ effizient abzubauen, wurden aus Pilzen, thermophilen Actinomyceten und Pflanzenkompost isoliert.

Thermostabile Polyesterhydrolasen, wie z. B. TfCut2 aus Thermobifida fusca, sind vielversprechende Biokatalysatoren für ein umweltfreundliches PET-Recycling (Abb. 2G). Für die enzymatische Hydrolyse von PET wurde amorphe PET-Folie mit einer Dicke von $250 \mu \mathrm{m}$ in Stücke von ca. $1,5 \mathrm{~cm}^{2}$ geschnitten, mit $1 \mathrm{nmol} / \mathrm{cm}^{2}$ gereinigter TfCut2 versetzt und bei $70{ }^{\circ} \mathrm{C}$ und $1.000 \mathrm{U} /$ min inkubiert. Zur Analyse wurden die PETChips einer Bestimmung des Gewichtsverlustes unterzogen. Ein Gewichtsverlust von PET von bis zu $22 \%$ der PET-Probe konnte nachgewiesen werden [5]. Für die Weiterverwendung von TA und EG in $\mathrm{P}_{4} \mathrm{SB}$ wurden dann im Enzymreaktor größere Mengen des Hydrolysats mit Umsätzen von bis zu 100 \% hergestellt.

\section{Verstoffwechselung von Plastikmonomeren}

P. putida KT2440 wird aufgrund seiner Stressresistenz und seines hochaktiven

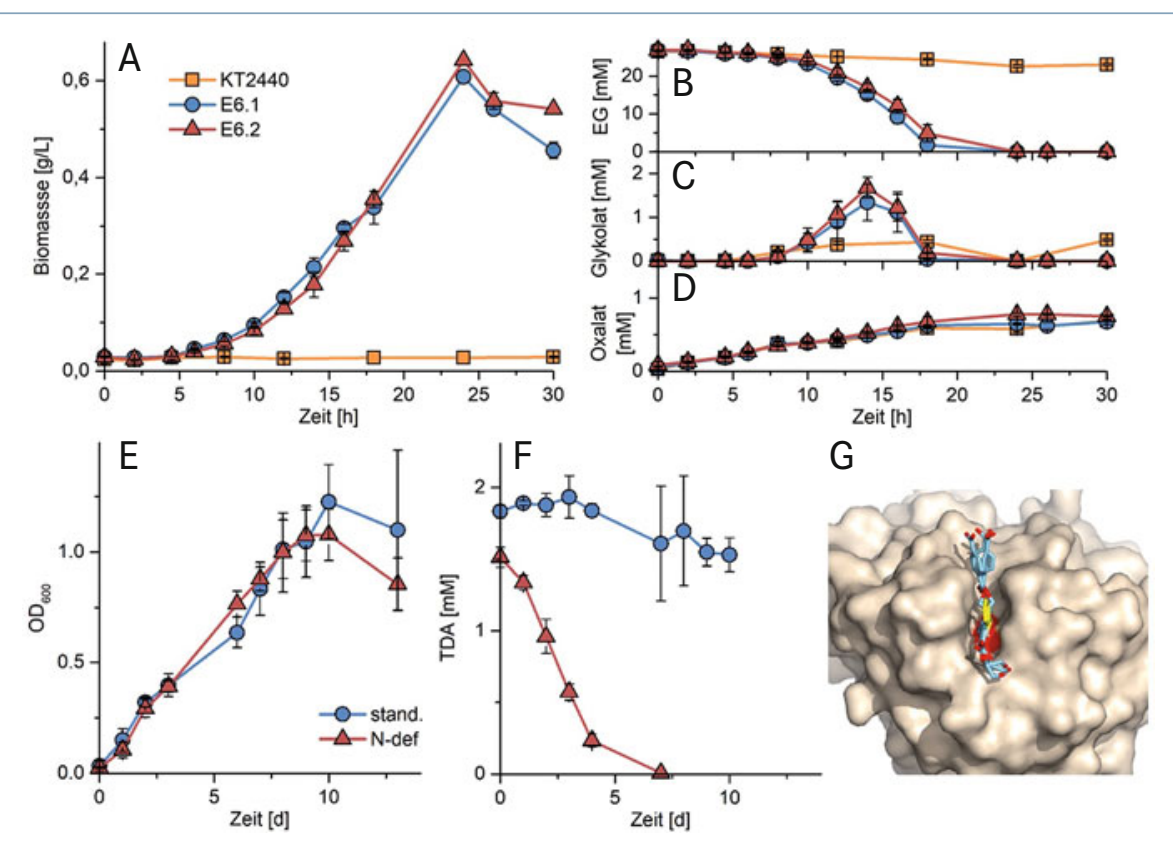

A Abb. 2: Wachstum auf Ethylenglykol (A) und Abbau von Ethylenglykol (EG) (B) von Pseudomonas putida KT2440 und zwei evolvierten Stämmen (E6.1 und E6.2). Außerdem ist die transiente Bildung von Glykolat (C) und die Akkumulation von Oxalat (D) gezeigt. E, Wachstum von Pseudomonas sp. TDA1 auf 2,4-Diaminotoluol (TDA) mit (blaue Kreise) und ohne zusätzliche Stickstoffquelle (rote Dreiecke). F, Abbau von TDA durch Pseudomonas sp. TDA1 (rote Dreiecke) und in einer sterilen Kontrolle (blaue Kreise). G, Polyesterhydrolase TfCut2 aus Thermobifida fusca mit einer PET-Modellverbindung in verschiedenen Konformationen in der Bindungstasche.

Redoxstoffwechsels zunehmend als Chassis für die industrieorientierte synthetische Biologie verwendet.

Biologischer Abbau von EG: Bei der biokatalytischen Depolymerisation von PET entstehen EG und TA in gleichen molaren Mengen. P. putida KT2440 ist nicht in der Lage, auf EG als einziger Kohlenstoffquelle zu wachsen. Adaptive Laborevolution (ALE) ist eine gängige Methode zur Anpassung von Stämmen an spezifische Umweltbedingungen. Um ein Wachstum von P. putida KT2440 auf EG zu erreichen, wurden drei parallele ALE-Kultivierungen in seriellen Satzkulturen mit EG als einziger Kohlenstoffquelle durchgeführt und nach dem Erreichen einer $\mathrm{OD}_{600}>0,5$ re-inokuliert [6]. Nach sechs seriellen Transfers wurden vereinzelte Stämme isoliert und im Growth Profiler (EnzyScreen, Heemstede, NL) hinsichtlich ihres Wachstums untersucht. Die Stämme E6.1 und E6.2 aus zwei parallelen ALE-Linien wurden für die weiteren Untersuchungen ausgewählt. In Minimalmedium mit $27 \mathrm{mM} \mathrm{EG}$ als alleiniger Kohlenstoffquelle wuchsen die Stämme mit einer Wachstumsrate von $0,19 \mathrm{~h}^{-1}$ (Abb. 2). Während des Wachstums auf EG konnten die Oxidationszwischenprodukte Glykolat (Abb. 2C) und Oxalat (Abb. 2D) im Medium gemessen werden. Die zugrunde liegenden genetischen Änderungen konnten aufgeklärt werden [6].

Biologischer Abbau von TDA/MDA: Aufgrund der großen Variabilität der Zusammensetzungen, ist bei den PU-Depolymerisationsprodukten eine große Vielfalt verschiedener Monomere $\mathrm{zu}$ erwarten. In $\mathrm{P}_{4} \mathrm{SB}$ ausgewählte PU-Modellpolymere waren EG, 1,4-Butandiol (BDO), Adipinsäure (AA) und die Abbauprodukte des Isocyanats, 4,4'-Diaminodiphenylmethan (MDA) und 2,4-Diaminotoluol (TDA).

Um ein Bakterium zu isolieren, das die aromatischen Diamine TDA und MDA als einzige Kohlenstoffquelle verwenden kann, wurde eine Plastikmülldeponie beprobt. Aus den Bodenproben konnte der Stamm TDA1 isoliert werden, der sowohl mit TDA und MDA als auch mit einem PU-Oligomer als alleiniger Kohlenstoffquelle wächst. Das Genom des isolierten Stammes wurde sequenziert und anhand des 16S-rRNA-Gens sowie seines Fettsäureprofils als Pseudomonas sp. identifiziert. Abb. 2E zeigt das Wachstum des TDA1-Isolats auf $2 \mathrm{mM}$ TDA. Die Wachstumsrate betrug $0,04 \mathrm{~h}^{-1}$, und TDA wurde vom Bakterium vollständig abgebaut (Abb. 2F). Mithilfe des sequenzierten Genoms und des Substratspektrums des iso- 


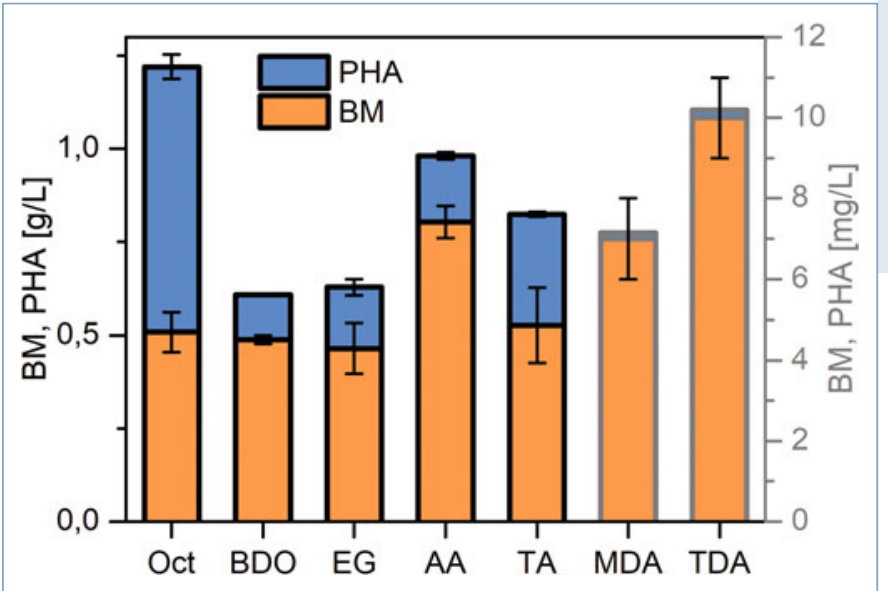

lierten Stammes wurde ein möglicher Abbauweg erarbeitet [7].

\section{Biosynthese von neuartigen Kunststoffen}

Für die PHA-Produktion aus den verschiedenen Plastikmonomeren wurden abhängig vom Substrat verschiedene PseudomonasStämme eingesetzt. Die Substrate BDO, AA und EG wurden von modifizierten P. putidaKT2440-Stämmen verstoffwechselt, während für den Abbau von TA, MDA und TDA Isolate (P. putida G016 und Pseudomonas sp. B2) eingesetzt wurden. In der Kontrolle wurde Oktanoat als Vergleichssubstrat verwendet. Die Konzentrationen der Substrate wurden so gewählt, dass die Kohlenstoffkonzentration bei $160 \mathrm{mM}$ lag. Allerdings musste diese bei MDA und TDA der Toxizität wegen auf $3 \mathrm{mM}$ verringert werden. Alle Substrate ermöglichten ein Wachstum der entsprechenden Stämme (Abb. 3). Allerdings konnte auf den Substraten MDA und TDA wegen der geringeren Substratkonzentration eine nur sehr geringe Biomassebildung beobachtet werden. Auch PHA konnte mit jedem Substrat produziert werden, wobei die höchsten Werte mit TA und AA erreicht werden konnten (ca. $30 \%$ der Biomasse bestand aus PHA).

4 Abb. 3: Produktion von Polyhydroxyalkanoaten (PHA), ausgehend von verschiedenen Plastikmonomeren durch Pseudomonas putida. Die linke $y$-Achse gilt für die linken fünf Balken, während die beiden rechten Balken die rechte $y$-Achse nutzen. BM steht für die Biotrockenmasse ohne PHA. Oct: Oktanoat; BDO: 1,4-Butandiol; EG: Ethylenglykol; AA: Adipinsäure; TA: Terephthalat; MDA: 4,4'-Diaminodiphenylmethan; TDA: 2,4-Diaminotoluol.

Mit diesen Ergebnissen sind wir der Vision von $\mathrm{P}_{4} \mathrm{SB}-$ einer stofflichen Nutzung von Plastikmüll - deutlich nähergekommen. Die Vielfalt von Plastikmonomeren und die damit einhergehende Freiheit im Design des Produktsyntheseweges wird eine Optimierung der Produktausbeute auf Substrat ermöglichen. Mit der im Klimapaket gestarteten $\mathrm{CO}_{2}$-Bepreisung und dem hier beschriebenen Upcycling wird die stoffliche Nutzung von Plastikmüll auch ökonomisch interessant.

\section{Danksagung}

Die Autoren bedanken sich für die Förderung durch die EU-Kommission im Rahmen vom Horizont 2020 Forschungs- und Innovationsprogramm (Projektnummer 633962, $\mathrm{P}_{4} \mathrm{SB}-$ From Plastic waste to Plastic value using Pseudomonas putida Synthetic Biology, www.p4sb. $\mathrm{eu})$.

\section{Literatur}

[1] Garcia JM, Robertson ML (2017) The future of plastics recycling. Science (New York) 358:870-872

[2] Geyer R, Jambeck JR, Law KL (2017) Production, use, and fate of all plastics ever made. Sci Adv 3:e1700782

[3] Jambeck JR, Geyer R, Wilcox C et al. (2015) Plastic waste inputs from land into the ocean. Science 347:768-771 [4] Wierckx N, Prieto MA, Pomposiello P et al. (2015) Plastic waste as a novel substrate for industrial biotechnology. Microb Biotechnol 8:900-903

[5] Wei R, Breite D, Song C et al. (2019) Biocatalytic degradation efficiency of postconsumer polyethylene terephthalate packaging determined by their polymer microstructures. Adv Sci 6:1900491

[6] Li W-J, Jayakody LN, Franden MA et al. (2019) Laboratory evolution reveals the metabolic and regulatory basis of ethyl- ene glycol metabolism by Pseudomonas putida KT2440. Environ Microbiol 21:3669-3682

[7] Cárdenas Espinosa MJ, Colina Blanco A, Schmidgall T et al. (2020) Towards biorecycling: isolation of a soil bacterium that grows on a polyurethane oligomer and monomer. Front Microbiol (akzeptiert)

\section{Funding: Open Access funding provided by Projekt DEAL.}

Open Access: Dieser Artikel wird unter der Creative Commons Namensnennung 4.0 International Lizenz veröffentlicht, welche die Nutzung, Vervielfälltigung, Bearbeitung, Verbreitung und Wiedergabe in jeglichem Medium und For
erlaubt, sofern Sie den/die ursprünglichen Autor(en) und die Quelle ordnungsgemäß nennen, einen Link Zur Creative Commons Lizenz beifügen und ordnungsgemäß nennen, einen Link zur Creative Commons Lizenz beifü
angeben, ob Änderungen vorgenommen wurden. Die in diesem Artikel angeben, ob Änderungen vorgenommen wurden. Die in diesem Artikel
enthaltenen Bilder und sonstiges Drittmaterial unterliegen ebenfalls der genannten Creative Commons Lizenz, sofern sich aus der Abbildungslegende nichts anderes ergibt. Sofern das betreffende Material nicht unter der genannten Creative Commons Lizenz steht und die betreffende Handlung nich nach gesetzlichen Vorschriften erlaubt ist, ist für die oben aufgeführten Weiterverwendungen des Materials die Einwilligung des jeweiligen Rechteinhabers einzuholen. Weitere Details zur Lizenz entnehmen Sie bitte der Lizenzinformation auf http://creativecommons.org/licenses/by/4.0/deed.de.

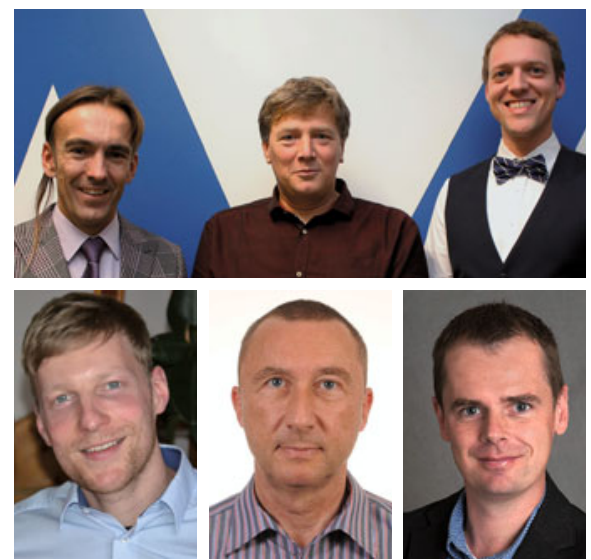

Lars M. Blank, Hendrik Ballerstedt, Till Tiso (v. I.n. r., oben) und Christian Eberlein, Wolfgang Zimmermann, Nick Wierckx (unten)

\section{Korrespondenzadresse:}

Prof. Dr. Lars M. Blank

Institute of Applied Microbiology - iAMB

Aachen Biology and Biotechnology - ABBt RWTH Aachen University

Worringerweg 1

D-52074 Aachen

lars.blank@rwth-aachen.de 